\title{
Research on the Artificial Acceleration Test System of Combined Environment and Loading Effect for the Bridge Structure ${ }^{+}$
}

\author{
Guowen Yao ${ }^{1}$, Xicheng Tan ${ }^{1}$, Shiya Li ${ }^{1, *}$, Peiyan Huang ${ }^{2}$ and Jiayu $\mathrm{Yu}^{1}$ \\ 1 State Key Laboratory Breeding Base of the Mountain Bridge and Tunnel Engineering, \\ Chongqing Jiaotong University, Chongqing 400074, China; yaoguowen@sina.com (Y.G.); \\ txc963266404@sina.com (T.X.); cqjtuyjy@163.com (Y.J.) \\ 2 School of Civil Engineering and Transportation, South China University of Technology, Guangzhou \\ 510641, China; pyhuang@scut.edu.cn \\ * Correspondence: lishiya@sina.com; Tel.: +86-23-6278-9146 \\ + Presented at the 18th International Conference on Experimental Mechanics (ICEM18), Brussels, Belgium, \\ 1-5 July 2018.
}

Published: 29 May 2018

\begin{abstract}
The bridges will be in service in a complex environment for over 100 years under the increasing traffic loading. So the long-term performance determines their safety and durability. Limited by the test condition and ability, present researches are mostly focused on the natural environment exposure tests and artificial environment acceleration tests for materials and components. According to the service conditions and load characteristics of the bridges, the accelerated test system was developed for bridge structure under combined environment and loading. This test system consists of three parts, including a large environment box, loading system and monitoring system. The environment box is $32 \mathrm{~m}$ long, $4 \mathrm{~m}$ wide and $3 / 10 \mathrm{~m}$ high. It is divided into 5 working bins which can be used alone or in combination. The simulation environment includes high temperature drying, low temperature freezing and thawing, damp cold, high temperature cycle, salt fog, rain, carbonation, temperature and humidity alternating and optical spectrum light aging environment. The servo static and dynamic loading system adopts Servotest actuators with loading tonnage from 400 tons to 50 tons. And the total tonnage is 1200 tons. Aided by the prestressed concrete reaction wall, steel structure frames and the anchor system, the external loading system can exert dynamic and static loads on the bridge structure in the environment box. And the non-contact nondestructive monitoring system can real-time monitor and measure their long-term deformation, internal force, cracking and other damage. This artificial acceleration test system can support the Chinese Long Term Bridge Program, and improve the safety, durability, reliability and long service life of bridges and other civil engineering.
\end{abstract}

Keywords: bridge structure; combined environment and loading effect; artificial acceleration test system; long term performance

\section{Introduction}

Under the coupling of environment and load, the long-term performance of the bridge structure is deteriorating. In order to study the durability of the bridge, the American Rom Airshow Center proposed an accelerated life test method in 1967. Limited by the experimental conditions and ability, the present researches are mostly focused on the natural environment exposure tests and artificial environment acceleration tests for materials and components. Few studies have been carried out on the combined effects of environment and loading on the bridge structures. Zhou Hao [1] studied the 
durability of RC truss beams reinforced with FRP-reinforced damp-heat. Qin Guang [2] studied the fatigue properties of CFRP-reinforced RC beams in hot and humid environment. Liu Hongwei [3] studied the chloride ion erosion in the marine environment. Song Wali [4] studied the effect of freezethaw cycles on the mechanical properties of concrete. The reason why the combined effects of the structure on the coupling of the environment and the load are rarely achieved is attributed to the limitations of the test equipment system. In Japan, the Material Systems and Technology Research Corporation has developed a simulation system for the damp-heat-vibration integrated environment, which realizes the coupling of temperature, humidity, and narrow-band with regular spectrum random loads. In China, the environmental chamber in Central South University can simulate the coupling of temperature, humidity, acid/alkali salt/atomization, rain shower and constant amplitude cycle. South China University of Technology has developed wet heat, temperature-salt spray, rain shower and dynamic load coupled loading system. At present, the environmental simulation system hasn't solved the problems which include low environmental indicators, no combination of multiple environmental factors, no medium and broadband random loads, and the single form of loading. The space and loading method of the environmental simulation experiment system established is limited, and the large-scale structures cannot be tested. Therefore, it is necessary to develop an artificial acceleration test system with coupling the environment and load for bridge structures.

\section{Design Scheme of Test System with Combined Environment and Loading}

Aiming to the service environment conditions and load characteristics of the bridge structure, an artificial acceleration test system was developed for the coupling effect of the bridge structure environment and load, and an artificial acceleration test method was proposed under the coupling of multi-factor environment and random load. The test system includes three parts of environment system, loading system and monitoring system. The stressed concrete reaction walls, steel structure reaction frames, and ground anchor systems around the environment box were served as external loading systems. The external loading system is connected with the force transmission device through the loading hole, and can exert dynamic and static load on the bridge structure inside the environment box. The design scheme of this test system is shown as Figure 1.

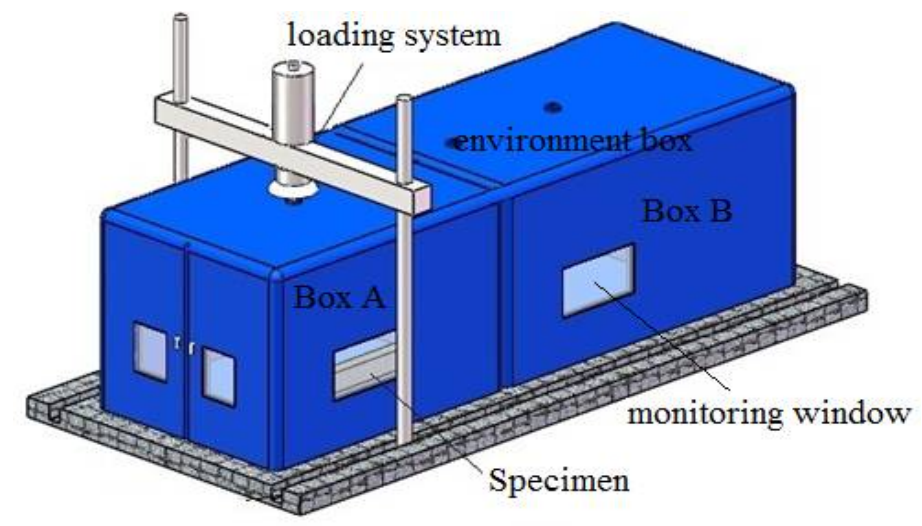

Figure 1. Design scheme of the test system with combined environment and loading.

\subsection{The Environment System}

The environment chamber is $32 \mathrm{~m}$ long, $4 \mathrm{~m}$ wide and 3 or $10 \mathrm{~m}$ high, it shown as Figures 2 and 3. It is divided into 5 cabins by movable doors. The smallest cabins $A$ and $B$ are $3 \mathrm{~m}$ long with space of 36 cubic meters. Cabin C is $6 \mathrm{~m}$ long. Cabins $\mathrm{D}$ and $\mathrm{E}$ are $10 \mathrm{~m}$ long, and cabin $\mathrm{E}$ is $10 \mathrm{~m}$ high. They can be used separately or combined. The box is sealed, insulated and anticorrosion, and connected with actuator by flexible sealing material. 


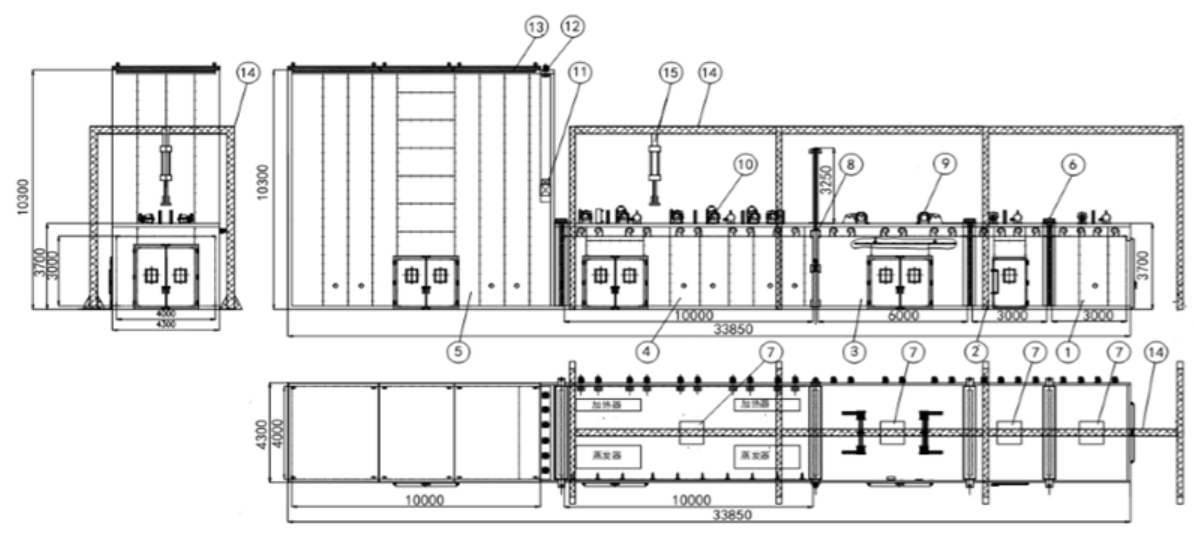

Figure 2. The large environment box with variable space.

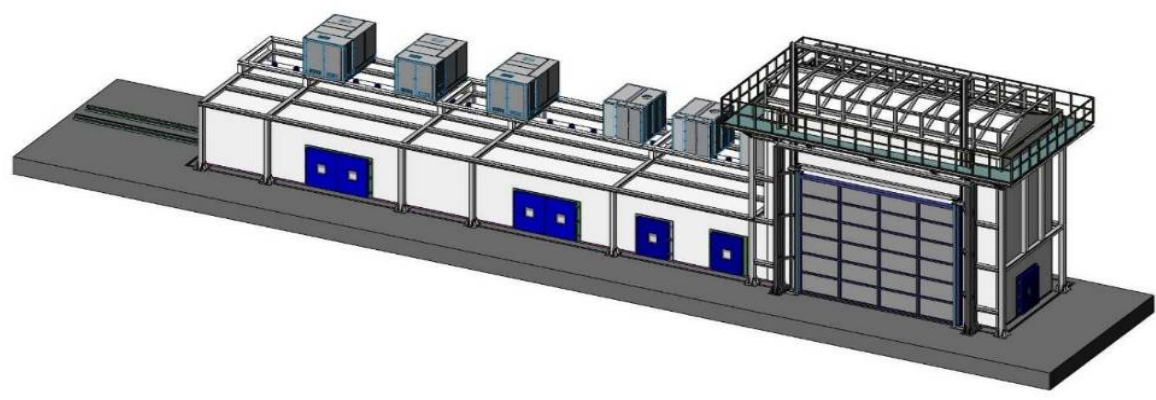

Figure 3. The large environment box with variable space.

The chamber can simulate various artificial acceleration environment, such as high temperature drying, low temperature freezing and thawing, high and low temperature cycling, salt spray, rainfall and temperature and humidity cycling. The temperature in cabin $\mathrm{A}$ is from $-70{ }^{\circ} \mathrm{C}$ to $100{ }^{\circ} \mathrm{C}$, and others are from $-40{ }^{\circ} \mathrm{C}$ to $100{ }^{\circ} \mathrm{C}$. The relative humidity is from $20 \%$ to $98 \%$. The rainfall is $20 \mathrm{~mm} / \mathrm{h}$ with 1000 cycles. The salt spray sedimentation is up to $5 \mathrm{~mL} / \mathrm{cm}^{2} * \mathrm{~h}$. Compared with conventional test, this system expands low temperature region, especially the low temperature and low humidity region. The range of temperature and humidity is shown as Figure 4.

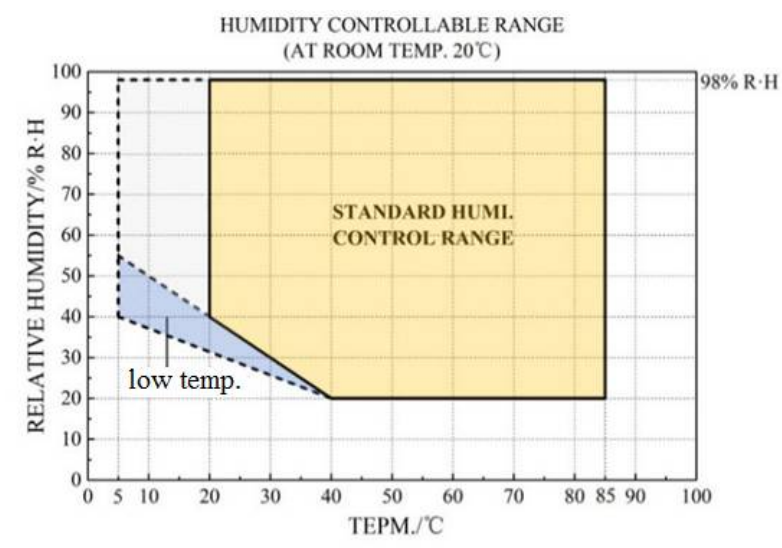

Figure 4. The range of temperature and humidity.

The chamber can be decomposed into refrigeration system, heating system, humidification system, dehumidification system, salt spray system, rainfall system and control system [5]. The system framework of the environment system is shown as Figure 5. The refrigeration system adopts the Italy DORIN piston compressor with water cooling and advanced hot gas bypass regulation technology, which significantly improves the efficiency and saves energy. The heating system adopts 
an efficient open-circuit electronic heater with double over-temperature protection and whole-time control by solid state relay. The humidification system provides steam by an external pressurecontrolled steam generator. The humidification speed is fast and the control precision is high. The dehumidification system adopts honeycomb wheels to absorb moisture repeatedly and provide dry air. Salt spray system can spray the corrosion solution. The control system adopts programmable controller with two running modes of constant temperature and humidity and alternation.

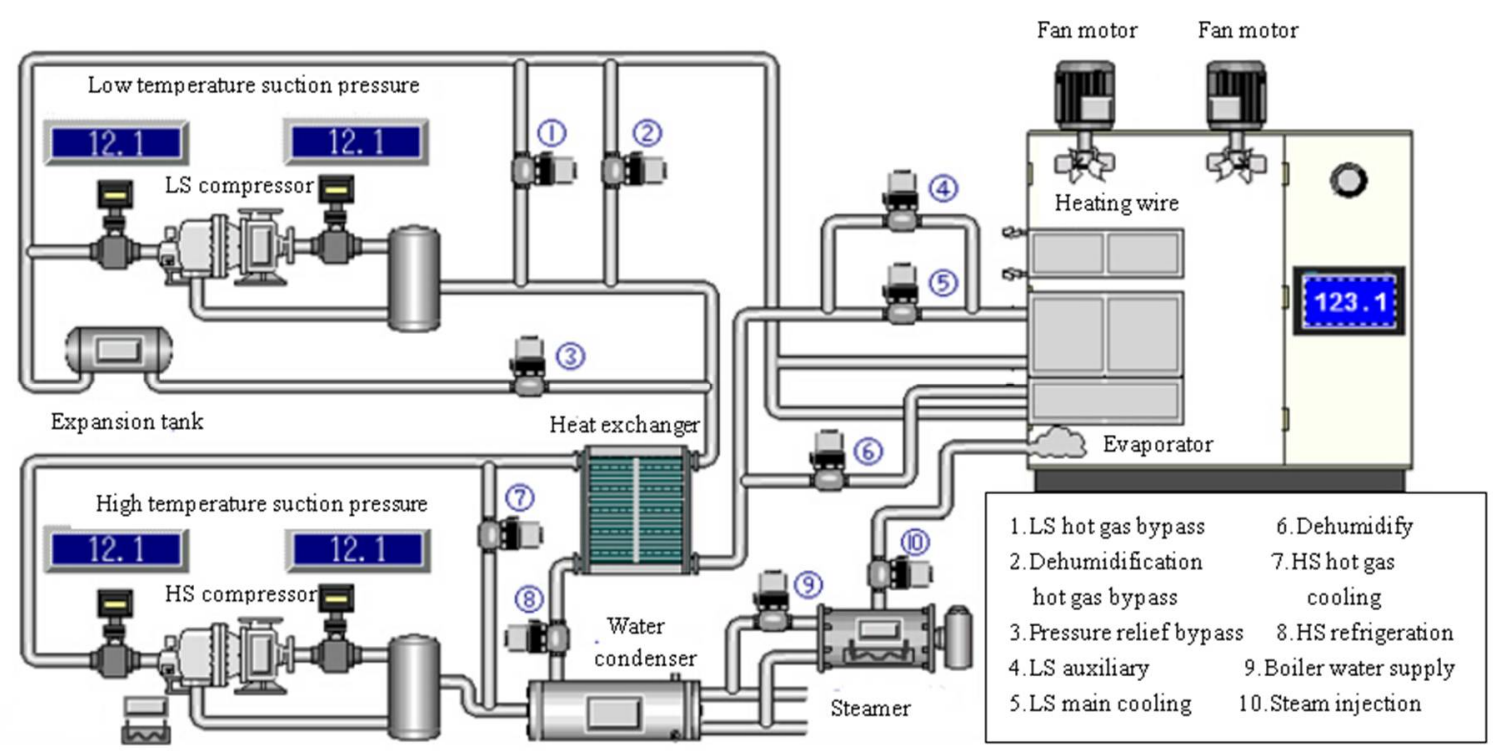

Figure 5. The framework of the environment system.

\subsection{The Loading System}

The large tonnage loading system is designed to study the failure process of the large bridge structures. It can conduct the static or dynamic test. The servo loading system adopts Servotest actuators, it shown as Figure 6, including 50, 100, 200, 400 tons, and the total tonnage of 1200 tons. There are 8 electro-hydraulic servo actuators with one or two-end out pole and $50 \mathrm{~cm}$ piston travel. The total flow of oil source is 750 liters per minute at $21 \mathrm{MPa}$ including 4 oil stations. The random load spectrum is simulated in the distribution and transformation method with medium or wide frequency.

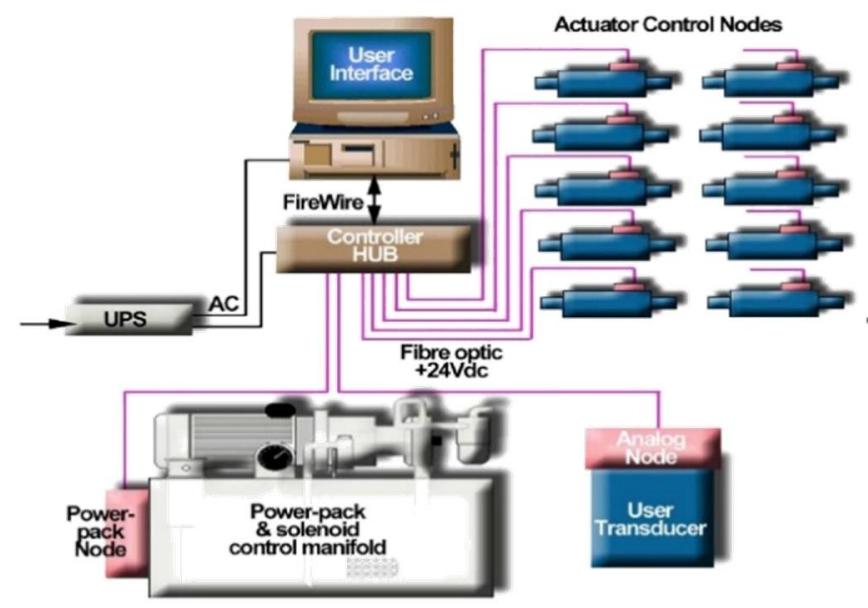

Figure 6. The loading system.

Bridges are usually subjected to random loads such as wind, rain, earthquakes, and vehicle loads. In order to be able to realistically study the fatigue of bridge structures, it is necessary to compile the load spectrum of fatigue experiments. It is necessary to collect data on the vehicles actually operating 
on the bridge and classify the vehicles by weight: trolleys, trucks, carts and trailers, and make classified vehicles-time series charts. According to the stochastic process theory, the statistical analysis of the traffic flow data can obtain the autocorrelation function and power spectral density function of the vehicle load sequence [6]. Using the distribution conversion method to complete the conversion of non-Gaussian to Gaussian process [7], the random load spectrum is transformed into a random load spectrum for fatigue testing. The random load spectrum can represent the load spectrum of actual conditions [8]. The parameters of the random load spectrum are input to the controller of the large-tonnage multi-functional servo dynamic and static loading test system, and the random load simulation can be achieved. The measured vehicle load spectrum through investigation and analysis is shown as Figure 7, and the experimental load spectrum of distribution conversion is shown as Figure 8.

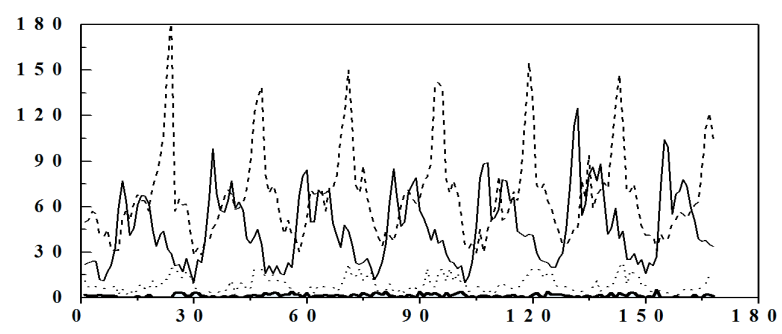

Figure 7. Measured vehicle load.

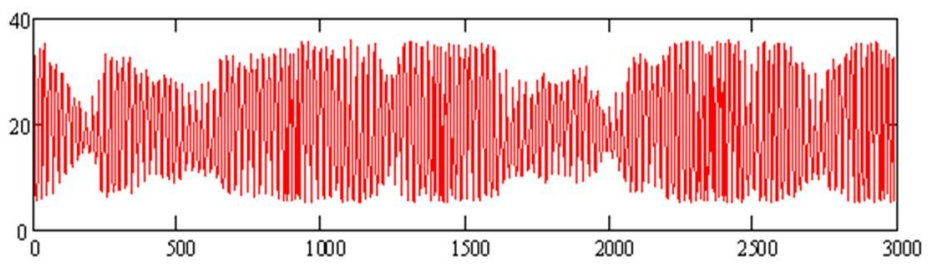

Figure 8. Experimental load spectrum.

The prestressed concrete reaction wall around the environmental box, the steel structure reaction rack and the ground anchor system exert dynamic and static load on the bridge structure inside the environmental box. The mobile multi-functional loading device can simulate fatigue loads.

\subsection{The Monitoring System}

The non-contact nondestructive monitoring system can real-time monitor and measure the deformation of the specimen in high humidity, rainfall or salt spray environment by remote camera with image processing. It also can monitor the crack initiation and propagation in DIC method [9].

\section{Artificial Acceleration Test Method}

In order to simulate the actual environment more accurately, the multi-factor environment and load coupling acceleration test method is a comparatively valuable method. The multi-factor environment and load coupling acceleration test is a practical application of the orthogonal test method to combine individual environmental factors in the environment for multiple individual factors and to scientifically arrange the coupling of multiple environmental factors and loads. Accelerated tests use more severe test conditions. This can study the long-term performance cracking mechanisms of bridge structures such as deformation, cracks, modes, and displacements in the service environment. It will be compared with the existing field long-term observations, provide conditional support for the Long Term Bridge Program, and ultimately improve the safety, durability, reliability, and long-term life of the bridges in China. The artificial acceleration test of bridge under combined environment and loading is shown in Figure 9. 


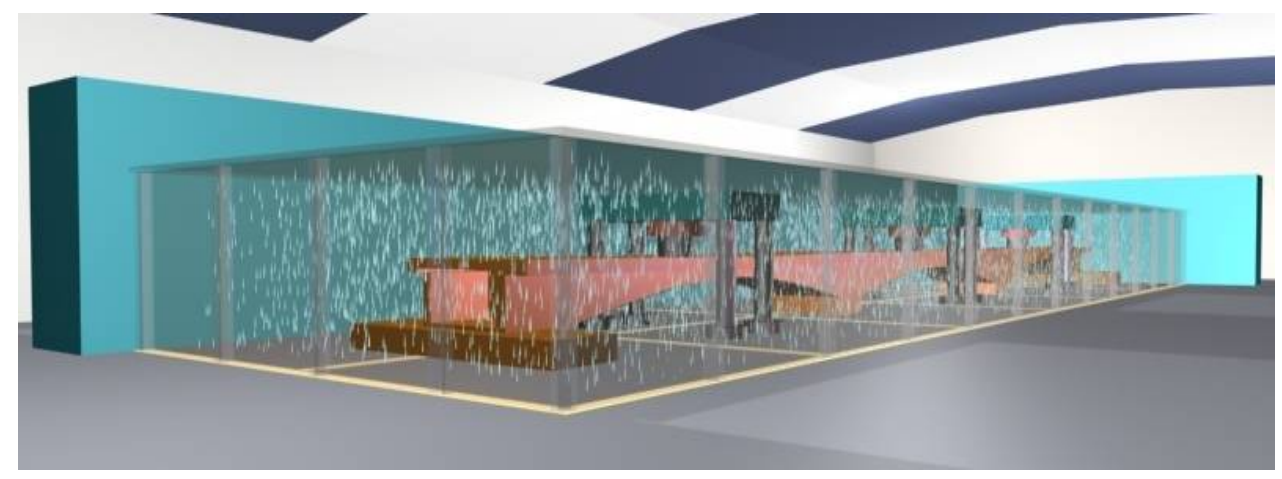

Figure 9. The artificial acceleration test of bridge under combined environment and loading.

\section{Conclusions}

An artificial acceleration test system was developed for the coupling effect of the bridge structure environment and load. An artificial acceleration test method was proposed under the coupling of multi-factor environment and random load. Test system includes the environment system, the loading system and the monitoring system. The test system can carry out coupling accelerated single or multi-factor environment and load tests for bridge structures. It is helpful to evaluate the reliability or remaining life of bridges under normal service environment and to lay a solid foundation for the health monitoring of bridges. This artificial acceleration test system can support the Chinese Long Term Bridge Program, and improve the safety, durability, reliability and long service life of bridges and other civil engineering.

Acknowledgments: This paper was supported by the Program of National Key R\&D Plan of China (Grant No. 2017YFC0806001), the Major National Natural Science Foundation for Research Instrument Development of China (Grant No. 11627802), the National Natural Science Foundation of China (Grant No. 51478071), the Key project of foundation and frontier research of Chongqing (Grant No. cstc2015jcyjBX0022), and the Science and Technology Research Project of Chongqing Education Commission (Grant No. KJ1600531).

\section{References}

1. Zhou, H. Research on Durability Experimental Method of RC Members Reinforced with FRP under Hot and Humid Environment. Doctor's Thesis, South China University of Technology, Guangzhou, China, 2012.

2. Qin, G.; Huang, P.; Zhou, H.; Guo, X.; Zheng, X. Fatigue and durability behavior of RC beams strengthened with CFRP under hot-wet environment. Constr. Build. Mater. 2016, 111, 735-742.

3. Liu, H.; Yang, X.; Li, J. Experimental study on erosion damage of chloride ion in concrete under the coastal beach environment. J. Yancheng Inst. Technol. 2017, 30, 14-17.

4. Song, W.; Li, X.; Ma, K. The effect of freeze-thaw cycles on mechanical properties of concrete. Adv. Mater. Res. 2011, 163-167, 3429-3432.

5. Li, W. Accelerated Test Method and Equipment System for Bridge Structure under Coupling Environment and Load. Master's Thesis, Chongqing Jiaotong University, Chongqing, China, 2015.

6. Yan, W.; Wan, H.; Ren, W. Analytical local and global sensitivity of power spectrum density functions for structures subject to stochastic excitation. Comput. Struct. 2017, 182, 325-336.

7. Atalay, K.D.; Tanyer, S.G. Randomness tests for the method of uniform sampling quasi-random number generator (MUS-QRNG). In Proceedings of the Signal Processing and Communications Applications Conference, Trabzon, Turkey, 23-25 April 2014; pp. 522-525.

8. Zheng, S.; Ju, F.; Feng, J. A new fatigue life prediction model by random load spectrum. J. Mech. Strength 2014, 36, 261-266.

9. Kondo, K.; Uchida, O.; Oda, K. Development of non-contact bridge beam deflection measurement technique with digital camera image sequence. J. Jpn. Soc. Photogramm. Remote Sens. 2016, 55, 90-94. 